\title{
Progress in hydrostatic extrusion of titanium
}

\author{
Krzysztof Topolski • Waclaw Pachla • \\ Halina Garbacz
}

Received: 31 October 2012/ Accepted: 10 December 2012/Published online: 20 December 2012

(C) The Author(s) 2012. This article is published with open access at Springerlink.com

\begin{abstract}
At the beginning of the present century it has been demonstrated that nanocrystalline titanium (NC-Ti) can be fabricated by hydrostatic extrusion (HE) which is one of the severe plastic deformation method. NC-Ti obtained in such a way exhibited excellent mechanical properties. The influence of $\mathrm{HE}$ on the microstructure and mechanical properties of titanium Grade 2 was first analyzed in 2005. In this study the progress in HE of titanium has been described. However, there are tribological and technological challenges encountered in HE of titanium. They can practically be eliminated by modifying the surface of Ti billets with aluminum coatings. Another important issue in extrusion is the optimum value of the accumulated strain necessary for grain refinement of titanium. Our results have shown that this value must exceed 3 to obtain nanocrystalline Ti. The results obtained indicate that HE permits producing NC-Ti rods with the diameter up to $10 \mathrm{~mm}$.
\end{abstract}

\section{Introduction}

The hydrostatic extrusion (HE) was invented over a 100 years ago and was patented by James Robertson in

K. Topolski $(\square) \cdot$ H. Garbacz

Faculty of Materials Science and Engineering,

Warsaw University of Technology, Woloska 141,

02-507 Warsaw, Poland

e-mail: kt.topolski@gmail.com

H. Garbacz

e-mail: hgarbacz@inmat.pw.edu.pl

W. Pachla

Institute of High Pressure Physics, Polish Academy of Science, Sokolowska 29/37, 01-142 Warsaw, Poland

e-mail:wacek@unipress.waw.pl
1893. However, the first experiments with this method were carried out by Percy W. Bridgman in the mid of previous century as reported in [1-3]. In the 1960s and 1970s Avitzur and Pugh contributed significantly to the development of this method [1, 2, 4, 5]. Among extruded materials there were also titanium and its alloys. However, at the end of the previous century the industrial importance of this technique started to decline.

At the beginning of the present century researchers at the Faculty of Materials Science and Engineering, Warsaw University of Technology and the Institute of High Pressure Physics, Polish Academy of Science, conducted the first experiments with $\mathrm{HE}$ of titanium with the aim to applying severe plastic deformation (SPD) and to refine grain size to the nanometric scale. It has been further shown that the grain boundary strengthening results in a significant increase of hardness, yield stress, and tensile strength of titanium [6-9]. These results motivated us to continue investigations and to develop the HE of titanium. Since that time the hydroextrusion method has been applied for the fabrication of nanocrystalline titanium (NCTi). The first results of the investigation of the influence of $\mathrm{HE}$ on the microstructure and mechanical properties of $\mathrm{Ti}$ Grade 2 were published in 2005 [6]. Nowadays, in spite of changes in material dimensions, hydroextrusion method is generally classified as a SPD method [10-13] and recognized as a method of grain refinement in Ti. The reason of this classification is the ability to induce SPD and possibility of grain refinement in the materials.

The improved mechanical properties of NC-Ti combined with biocompatibility, low weight, and good corrosion resistance make it a very attractive and perspective material. However, there are still some remaining problems and challenges related to the adhesion of titanium to the material of the die, large surface development of the NC-Ti 
rods, and high pressure necessary for the extrusion. Another important issue for explanation and optimization is the value of accumulated strain necessary for grain refinement of titanium especially in the context of processing large volume products [10]. All these issues are discussed in the present paper which describes recent progress in $\mathrm{HE}$ of titanium.

\section{Material, processing, and investigation methods}

The material investigated was commercially pure titanium Grade 2 ( $\mathrm{Ti} \geq 99.38 \%$ mass) in the form of rods. The research program consisted of hydroextrusion processes of billets with various diameters $(12,20,33$, and $50 \mathrm{~mm})$. The diameter of the final NC-Ti product was between 3 and $10 \mathrm{~mm}$. HE of titanium is a multi-pass process conducted with the gradual reduction of the rod diameter typically in ten passes of extrusion. The extrusions were carried out at the Institute of High Pressure Physics, Polish Academy of Sciences, within the framework of a project coordinated by the Faculty of Materials Science and Engineering, Warsaw University of Technology. All the extrusion experiments were conducted at ambient temperature.

The nanostructures of the extruded samples were examined by transmission electron microscopy (TEM Jeol 1200). Analyses and observations of aluminum coatings were conducted using Hitachi 2600N and Hitachi TM-1000 scanning electron microscopes (SEM + EDS). All the tensile tests were carried out at room temperature and at the same initial straining rate $\hat{\varepsilon}=3.3 \times 10^{-4} \mathrm{~s}^{-1}$. The microhardness of the samples was measured on cross sections using the Vickers method under a load of $200 \mathrm{~g}$.

\section{Principle and description of the hydrostatic extrusion method}

The principle of the HE method is shown in Fig. 1. The medium (3) fills the chamber (5) and surrounds the billet (1). When the piston (4) moves, the medium is compressed and thus the hydrostatic pressure in the chamber increases. When the pressure becomes sufficiently high, deformation begins. The surface of the contact between the billet (1) and the die (6) is a zone of intensive friction and adhesion.

Characteristic features of hydroextrusion are: (a) no direct contact between the piston and the billet; (b) no direct contact between the billet and the chamber walls; and (c) the presence of a liquid medium inside the chamber. Hence the friction force is significantly reduced. Compared to the conventional extrusion and other deformation techniques, HE ensures better deformability of the material, which is due to the applied hydrostatic pressure.

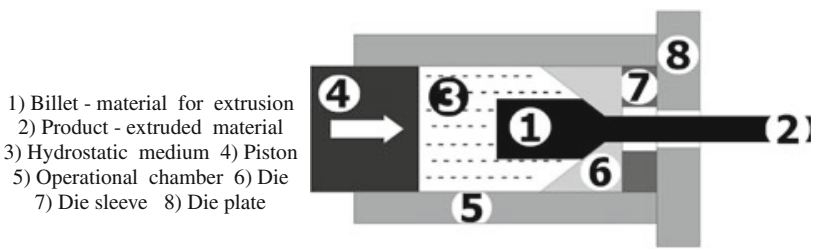

Fig. 1 The schematic representation of the hydrostatic extrusion (HE) method.

The hydroextrusion induces a uniform triaxial stress state. It makes the formation and propagation of cracks more difficult thereby increasing significantly the plasticity of the material.

\section{Surface modification}

The basic technological problems and challenges in the extrusion of titanium are:

(a) adhesion between titanium and the material of the die (resulting in a high friction force and wear of the die),

(b) large surface roughness of the NC-Ti rods (including scratches),

(c) high pressure needed for the extrusion (which restricts possibility of extrusion with larger deformation during one pass of extrusion).

The original results obtained show that by modification of the titanium surface with $10 \mu \mathrm{m}$ aluminum coatings these problems can be practically eliminated. Titanium rods were covered with aluminum coatings using the magnetron sputtering (MS) method. The assumption underlying this experiment was based on the concept of coatings with very good plasticity and adhesion to the substrate. The results of testing confirmed that this assumption was fulfilled. Comparing with microhardness results of $\mathrm{Ti}$ base $\left(\mathrm{HV}_{0.01}=342\right)$ the microhardness of $\mathrm{Al}$ coating was much lower $\left(\mathrm{HV}_{0.01}=202\right)$. Using scratch test any cracks or spalling were not visible. The aluminum coating had an excellent plasticity. The examinations using scanning electron microscopes show that the $\mathrm{Al}$ coatings are homogeneous and distributed uniformly (Fig. 2). They have the adhesive character and do not contain the intermetallic $\mathrm{Ti}-\mathrm{Al}$ transition phases of the diffusive character [energy dispersive spectroscopy (EDS) analysis-Fig. 3].

During the extrusion process, the $\mathrm{Al}$ coating and $\mathrm{Ti}$ substrate were subjected to plastic deformation. Despite the strain, the $\mathrm{Al}$ coatings maintain good adhesion to the titanium substrate with no visible cracking or spalling. The results proved that the coatings functioned as an excellent lubricant which separated the titanium rods from the die. This reduced the friction and protected titanium from 


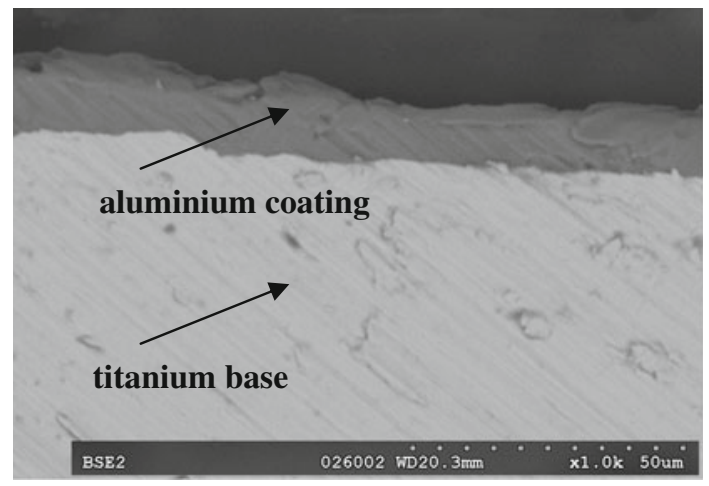

Fig. 2 SEM image of an Al coating (10 $\mu \mathrm{m}$ thick) formed on a $\mathrm{Ti}$ substrate before $\mathrm{HE}$

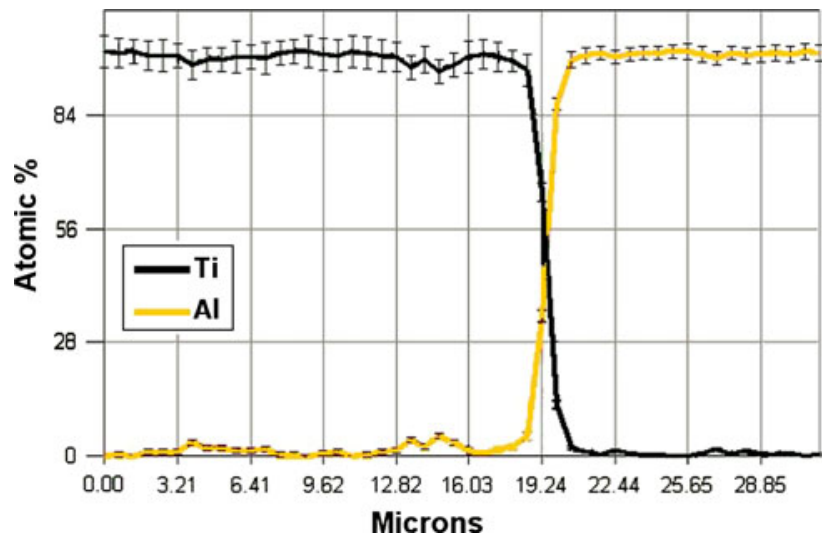

Fig. 3 EDS analysis of an $\mathrm{Al}$ coating $(10 \mu \mathrm{m}$ thick) and its $\mathrm{Ti}$ substrate before HE

adhering to the die. Also, based on the macroscopic observations, the surface of the extruded coated products is less rough. Another important advantage is a significant decrease of the extrusion pressure which permits increasing the strain applied in a single extrusion pass and deforming titanium with higher reduction of the rod diameter. This is a great profit which permits to decrease the number of passes necessary to produce NC-Ti. This effect is demonstrated in Fig. 4 which shows that reduction of the diameter $(D)$ of Ti rods from $\emptyset 12$ to $\emptyset 3 \mathrm{~mm}$ requires from 5 to 11 extrusion passes (fragments of the diagram between the individual points), whereas the Al-coated rods can be extruded in only two HE passes (Ø12-Ø6 and Ø6-Ø3) conducted at moderate pressure.

The benefits and progress in $\mathrm{HE}$ of $\mathrm{Ti}$ achieved due to the aluminum coatings are the following:

- reduction of the frictional wear of the die,

- decrease of the surface roughness of the extruded rods,

- significant reduction of the maximum extrusion pressure $\left(p_{\max }\right)$ permitting a higher reduction of the rod diameter during one extrusion pass,

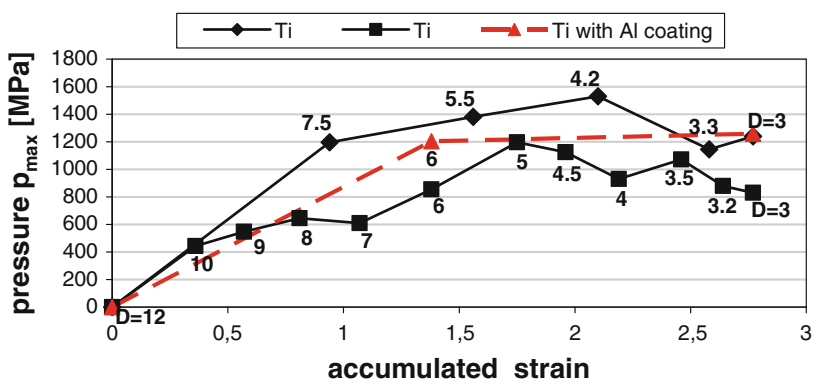

Fig. 4 Reduction of the number of extrusion passes achieved due to the presence of $\mathrm{Al}$ coatings. Maximum pressure as a function of accumulated strain during successive extrusion passes (where $D$ diameter of rods)

- decrease of the number of the extrusion passes needed for grain refinement.

\section{Accumulated strain}

The hydroextrusion of titanium is conducted in several passes in which the rod diameter is gradually reduced. During each pass the plastic deformation is induced into material. The sum of deformations induced by all the extrusion passes is defined as accumulated strain, which is one of the basic parameters of the extrusion process. It can be calculated from the formula:

$\varepsilon=2 \ln \left(D_{\mathrm{s}} / D_{\mathrm{f}}\right)$

where $D_{\mathrm{s}}$ is starting diameter and $D_{\mathrm{f}}$ is final diameter of the rod.

The HE of titanium may be conducted to obtain a wide range of accumulated strain. The aim of our experiments was to optimize the extrusion process by finding the minimum value of strain ensuring the formation of nanostructured titanium. It was also important to examine how the successive passes affect the structure and mechanical properties of titanium. The optimization of the accumulated strain will permit producing nano-titanium with the desired optimum mechanical properties and will permit avoiding an extra costs.

These experiments included 14 extrusion passes with gradual reduction of the rod diameter up to accumulated strain $\varepsilon=4.60$.

Our investigations have shown that the accumulated strain is the key parameter that decides about the strengthening of titanium and the formation of its nanostructure. We can see that the structure of titanium extruded with $\varepsilon=2.54$ features very high density of dislocations which form cells (Fig. 5). Nevertheless the nanostructure was not observed. Only a few nano-grains were observed occasionally, so the evaluation of average 


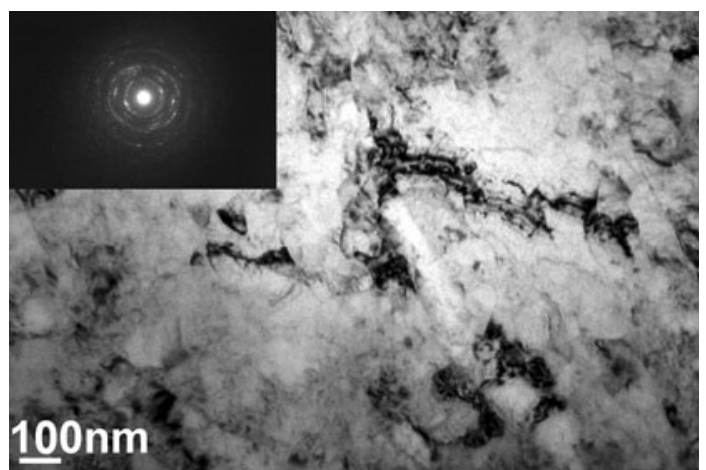

Fig. 5 TEM image of titanium extruded at accumulated strain $\varepsilon=2.54$

grain size was impossible. On the other hand, for the titanium extruded with accumulated strain $\varepsilon \geq 3.22$ the nanostructure was obtained as seen in TEM images and diffraction patterns (Figs. 6, 7, 8). For the accumulated strain between 3.22 and 3.93 , the grain size is in the range $73-62 \mathrm{~nm}$. The further increase of the strain to 4.60 does not change the size of the nanograins $\left[E\left(d_{2}\right)=62 \mathrm{~nm}\right.$-Fig. 8]. At higher strains, the refinement process is much slower and the grain sizes tend to a certain limiting value.

If the accumulated strain 2.5 is not sufficiently high for the formation of a nanocrystalline structure in titanium, whereas 3.2 is high enough, the present authors assumed that hydroextrusion guarantee nanocrystalline structure in titanium when the accumulated strain exceeds 3.0. Accumulated strain within the range from 3.2 to 4.6 does not change the average sizes of the nano-grains, but increases their number and their share in the volume of the material. The grain size distribution of NC-Ti obtained for strain 3.2 and 4.6 is similar also (Figs. 9, 10). With a high probability we can anticipate that the strain above 4.6 will give no further marked refinement and, thus, it is economically unjustified.

Extrusion with various accumulated strains permitted us to estimate its effect on the mechanical properties of titanium. The results are given in Table 1. It can be seen that with increasing strain the material is gradually strengthened. The greatest increase of yields stress (YS), ultimate tensile strength (UTS), and hardness (HV) was observed in the first stage of the experiment when the dislocation density in the material was lower. At the accumulated strain above 2.5 the strengthening of the material is weaker or does not occur at all. This is especially true in the case of YS and hardness. The UTS on the other hand increases almost linearly (Fig. 11).

The knowledge of how YS, UTS, and HV depend on the accumulated strain permits designing the material

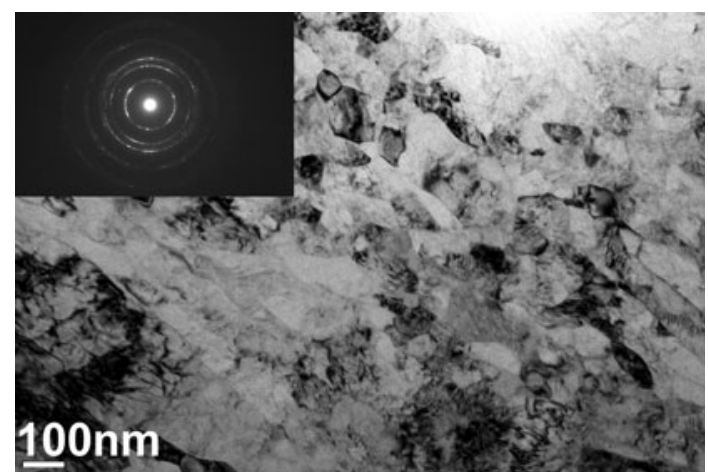

Fig. 6 TEM image of titanium extruded at accumulated strain $\varepsilon=3.22$

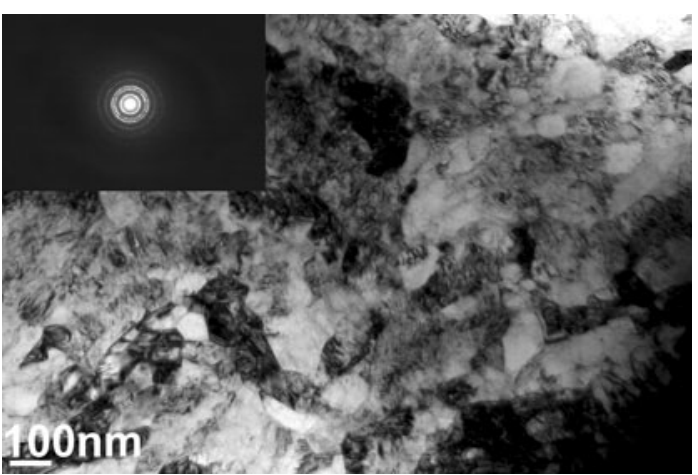

Fig. 7 TEM image of titanium extruded at accumulated strain $\varepsilon=3.93$

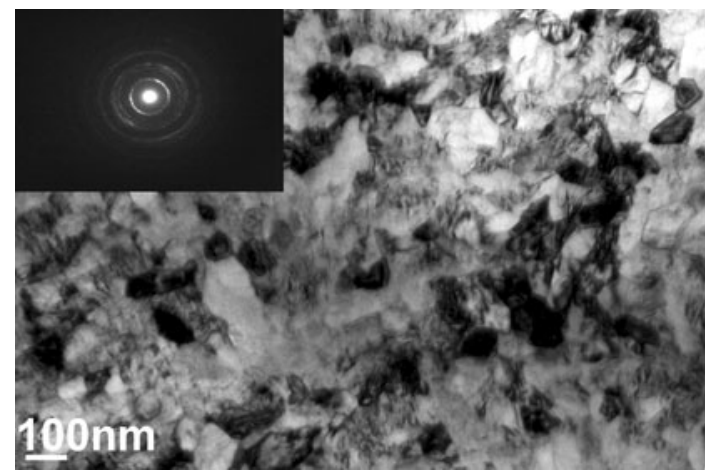

Fig. 8 TEM image of titanium extruded at accumulated strain $\varepsilon=4.60$

according to the current needs. It is noteworthy that the strength of the material extruded with the accumulated strain of 3.0 is similar to that of titanium alloys [14].

The authors of Ref. [13] carried out experiments with extrusion of titanium using the accumulated strain above 4.6, up to 5.4. For strain 5.4 the average grain size they obtained was $47 \mathrm{~nm}$ and the tensile strength of the material 
was $1320 \mathrm{MPa}$. Taking into account that, comparing to the samples with strain 4.6, the UTS increased by only $11 \%$, we can conclude that the extrusion with so high accumulated strain is less effective.

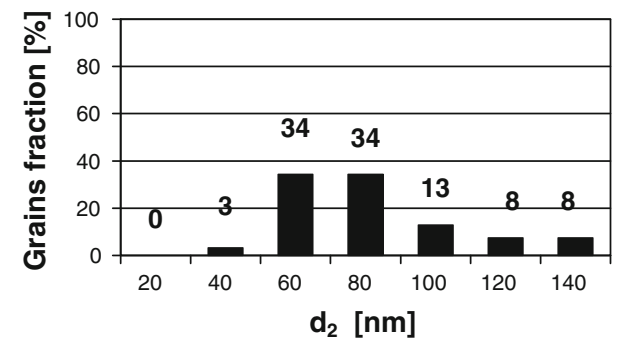

Fig. 9 Grain size distribution for sample after extrusion with accumulated strain 3.22

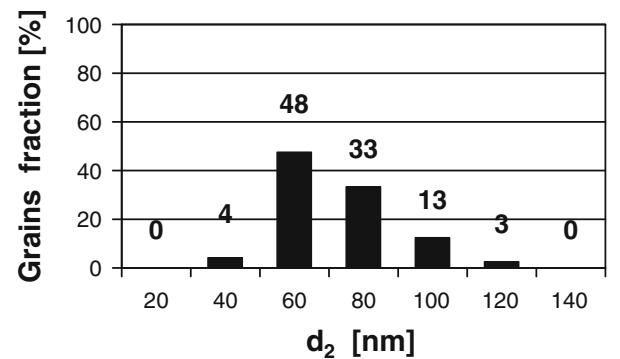

Fig. 10 Grain size distribution for sample after extrusion with accumulated strain 4.60

Table 1 Mechanical properties of titanium depending on the accumulated strain

\begin{tabular}{llllllll}
\hline$\varepsilon$-strain & 0 & 0.83 & 1.38 & 2.54 & 3.21 & 3.93 & 4.60 \\
\hline YS [MPa] & 357 & 614 & 736 & 878 & 860 & 1033 & 1045 \\
UTS [MPa] & 482 & 677 & 811 & 955 & 1053 & 1144 & 1190 \\
A [\%] & 21.1 & 6.8 & 6.8 & 6.0 & 8.1 & 6.8 & 6.9 \\
HV $_{0.2}$ & 184 & 222 & 237 & 242 & 266 & 256 & 279
\end{tabular}

$Y S$ yield stress, UTS ultimate tensile strength, $A$ elongation, $H V_{0.2}$ microhardness

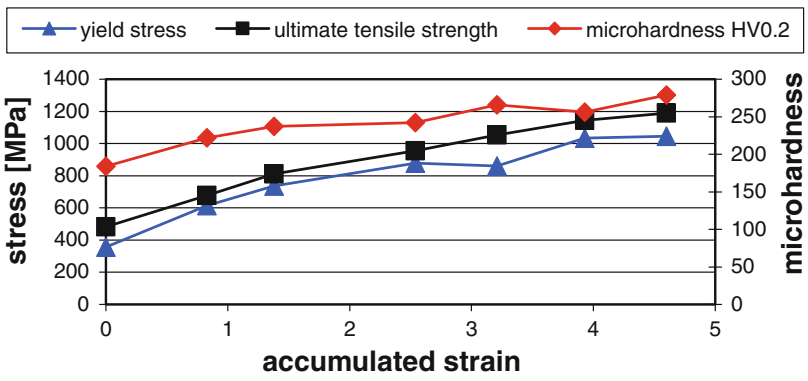

Fig. 11 Dependence of YS, UTS, and HV on accumulated strain

\section{Volume of NC-Ti}

In producing nano-metals one of the basic challenges is to obtain a sufficiently large volume of the final material. This is also important with NC-Ti developed for medical applications [15-17].

During our first experiments with refining titanium microstructure by HE we achieved the nano-titanium in the form of a rod $3 \mathrm{~mm}$ in diameter $[12,18]$. In the next years we attempted to produce nano-products with larger volume and we tried to examine the possibility of scaling up the hydroextrusion of titanium.

The experiments with the increasing diameter of the nanoproduct were successful. We obtained NC-Ti rods with larger diameters such as 5, 7, and $8 \mathrm{~mm}$ (Fig. 12) [19]. Finally, we managed to produce an NC-Ti rod with a diameter of $10 \mathrm{~mm}$ (Fig. 13). The material was very homogeneous along the length and along the diameter [20]. All the NC-Ti rods had similar grain sizes below $100 \mathrm{~nm}$ (Table 2). The typical length of these rods was about $250-300 \mathrm{~mm}$.

The results of our experiments have shown that by accumulating strain above 3.0 it is possible to refine its structure to the nano-scale, irrespective of the billet or product diameter. Products of larger diameters can be

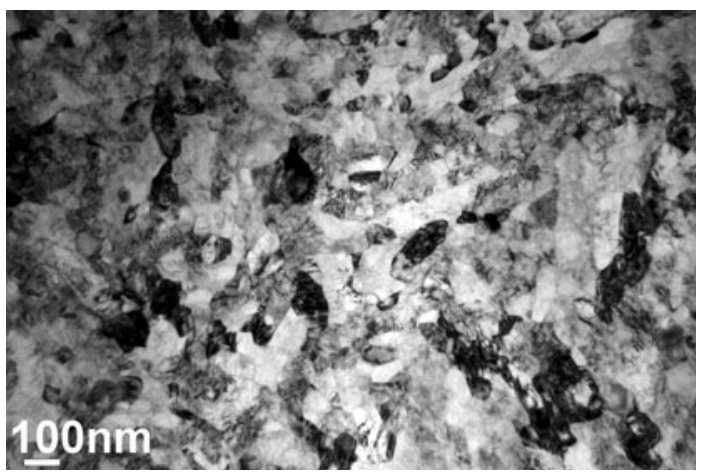

Fig. 12 Nanocrystalline titanium in the form of a rod $5 \mathrm{~mm}$ in diameter

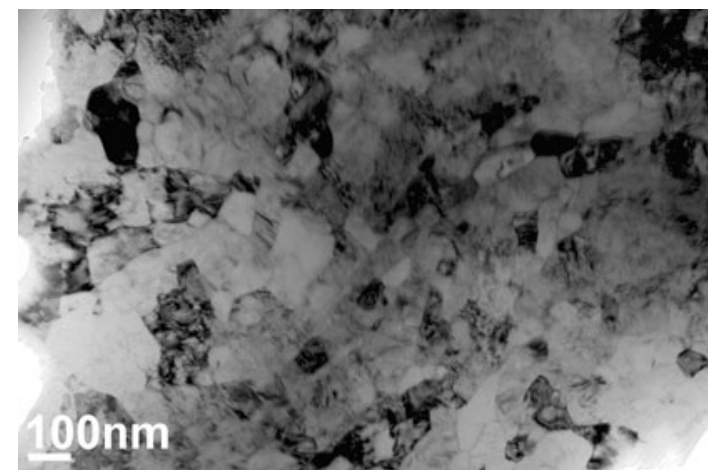

Fig. 13 Nanocrystalline titanium in the form of a rod $10 \mathrm{~mm}$ in diameter 
Table 2 Average size $E\left(d_{2}\right)$ of the nano-grains and mechanical properties of final bulk NC-Ti

\begin{tabular}{lrrr}
\hline NC-Ti rod diameter $[\mathrm{mm}]$ & \multicolumn{1}{c}{8} & \multicolumn{1}{c}{10} \\
\hline$E\left(d_{2}\right)[\mathrm{nm}]$ & 55 & 59 & 73 \\
YS $[\mathrm{MPa}]$ & 1040 & 960 & 860 \\
$\mathrm{UTS}[\mathrm{MPa}]$ & 1141 & 1069 & 1053 \\
$\mathrm{HV}_{0.2}$ & 273 & 273 & 266 \\
\hline
\end{tabular}

produced by increasing the diameter of the billet. This opens the possibility of scaling up the process of hydroextrusion of titanium. The only restriction is the construction and possibility of the installation.

The product with increased volume had very good mechanical properties similar to the NC-Ti obtained in our earlier experiments (Table 2). It should be noted that, compared with many typical titanium alloys, the mechanical properties of NC-Ti obtained in our experiments were similar or superior $[14,21,22]$.

\section{Conclusions}

The last 10 years of experiments and studies have resulted in a significant progress in processing NC-Ti by HE. The HE method applied to titanium has been successfully developed and optimized.

Modification of titanium surface with aluminum coatings improved the tribological conditions of the hydroextrusion process. It permitted reducing the frictional and adhesive wear of the die and the roughness of the surface of the product. The precision of shaping the product also increased. Owing to the aluminum coating, the extrusion pressure could be lower which allowed increasing strain applied in one extrusion pass, and thereby, when processing $\mathrm{NC}-\mathrm{Ti}$, decreasing the number of the extrusion passes.

To obtain NC-Ti using the HE method at room temperature the accumulated strain should exceed a value of 3.0. This requirement is valid irrespective of the diameter of the billet. Our experiments have shown that it is possible to produce $\mathrm{NC}-\mathrm{Ti}$ in the form of rods with a diameter from 3 up to $10 \mathrm{~mm}$. Therefore, the possibility of scaling up the HE method has been confirmed.
Acknowledgements The studies were conducted at the Faculty of Material Science and Engineering at Warsaw University of Technology in the Project No. 507226440 financed by National Science Centre.

Open Access This article is distributed under the terms of the Creative Commons Attribution License which permits any use, distribution, and reproduction in any medium, provided the original author(s) and the source are credited.

\section{References}

1. Pugh HLD (1970) Mechanical behaviour of materials under pressure. Elsevier Publishing Company Limited, Amsterdam

2. Pugh HLD, Donaldson CJH (1972) Ann CIRP 21(2):167-186

3. Lange K (1985) Handbook of metal forming. McGraw-Hill Book Company, New York

4. Avitzur B (1965) Trans ASME J Eng Ind 87:487-494

5. Avitzur B, Sortais HC (1966) Trans ASME J Eng Ind 88:659-668

6. Dutkiewicz J, Kuśnierz J, Maziarz W, Lejkowska L, Garbacz H, Lewandowska M, Dobromyslov AV, Kurzydlowski KJ (2005) Phys Status Solidi A 202:2309

7. Garbacz H, Lewandowska M, Pachla W, Kurzydlowski KJ (2006) J Microsc 223:272

8. Pachla W, Kulczyk M, Swiderska-Sroda A, Lewandowska M, Garbacz H, Mazur A, Kurzydłowski KJ (2006) Proceedings of the 9th international ESAFROM conference on material forming, University of Strathclyde, Glasgow, pp 535-538

9. Kurzydlowski KJ (2006) Mater Sci Forum 503-504:341

10. Kurzydlowski KJ, Lewandowska M (2007) Mater Sci Forum 561-565:913

11. Lewandowska M, Kurzydlowski KJ (2008) J Mat Sci 43:7299. doi:10.1007/s10853-008-2810-z

12. Topolski K, Garbacz H, Kurzydlowski KJ (2008) Solid State Phenom 140:191

13. Pachla W, Kulczyk M, Sus-Ryszkowska M, Mazur A, Kurzydlowski KJ (2008) J Mater Process Technol 205:173

14. Niinomi M (1998) Mater Sci Eng A243:231

15. Valiev RZ, Semenova IP, Jakushina E, Latysh VV, Rack H, Lowe TC, Petruzelka J, Dluhos L, Hrusak D, Sochova J (2008) Mater Sci Forum 584-586:49

16. Valiev RZ (2006) Solid State Phenom 114:7-18

17. Latysh V, Krallics Gy, Alexandrov I, Fodor A (2006) Curr Appl Phys 6:262

18. Topolski K, Garbacz H, Kurzydlowski KJ (2008) Mater Sci Forum 584-586:777

19. Topolski K, Garbacz H, Pachla W, Kurzydlowski KJ (2010) Phys Status Solidi C7(5):391

20. Topolski K, Garbacz H, Pachla W, Kurzydlowski KJ (2011) Mater Sci Forum 674:47

21. Long M, Rack HJ (1998) Biomaterials 19:1621

22. Wang K (1996) Mater Sci Eng A213:134 